\title{
Neurocardiogenic injury in subarachnoid hemorrhage: A wide spectrum of catecholamin-mediated brain-heart interactions
}

\author{
Maciej Wybraniec ${ }^{1}$, Katarzyna Mizia-Stec ${ }^{1}$, Łukasz Krzych ${ }^{2}$ \\ ${ }^{1} 1^{\text {st }}$ Department of Cardiology, Medical University of Silesia, \\ Upper Silesian Medical Center, Katowice, Poland \\ ${ }^{2} 1^{\text {st }}$ Department of Cardiac Surgery, Medical University of Silesia, \\ Upper Silesian Medical Center, Katowice, Poland
}

\begin{abstract}
Background: The purpose of this review was to summarize the up-to-date knowledge on clinical presentation and management of neurocardiogenic injury and to deliver the evidence of common pathophysiology of this broad spectrum of disorders.

Methods: Medline and EmBase databases were searched to obtain original research articles and review papers using the following key words: neurocardiogenic injury, stress cardiomyopathy, tako-tsubo, subarachnoid hemorrhage, ECG abnormalities, catecholamine toxicity, neuropulmonary edema.

Results: Various forms of cerebral pathology, most importantly subarachnoid hemorrhage (SAH), are accompanied by transient cardiac dysfunction with ST-segment elevation and QT interval prolongation and $T$ wave inversion with simultaneous release of cardiac troponin. In the past 20 years a great deal of data emerged concerning stress cardiomyopathy ('tako-tsubo') presenting as a rare transient apical ballooning syndrome following stressful life events with symptoms and signs resembling acute myocardial infarction (AMI), yet without significant coronary artery stenosis. Both forms of cardiac dysfunction are mediated by catecholamine toxicity, triggered by physical and psychological distress, leading to a specific type of neurogenic myocardial stunning reflected by histopathological image of contraction band necrosis.
\end{abstract}

Conclusions: Neurocardiogenic injury should be carefully differentiated from AMI. Cardiac dysfunction in SAH heralds increased mortality. The criteria for the diagnosis of stress cardiomyopathy should be revised to comprise the diversity of its clinical symptomatology and to include cardiac dysfunction accompanying cerebral pathology. (Cardiol J 2014; 21, 3: 220-228)

Key words: neurocardiogenic injury, stress cardiomyopathy, tako-tsubo, subarachnoid hemorrhage, ECG abnormalities

Address for correspondence: Maciej Wybraniec MD, $1^{\text {st }}$ Department of Cardiology, Medical University of Silesia, ul. Ziołowa 47, 40-635 Katowice, Poland, tel: +48 3235986 11, fax: +48 3225270 66, e-mail: wybraniec@os.pl Received: 22.03.2013 Accepted: 06.06.2013 


\section{Introduction}

In the setting of an emergency department, clear differentiation between acute myocardial infarction (AMI) and other acute conditions requiring prompt management is of vital importance. Various neurological disorders, most importantly subarachnoid hemorrhage (SAH), were reported to have an electrographic presentation resembling characteristic features of AMI in the form of repolarization abnormalities (e.g. ST-segment elevation) [1]. Commonly considered misleading and nonspecific, symptoms of SAH can sometimes be almost indistinguishable from those of AMI [2], which often leads to initial coronary angiography revealing no coronary artery disease (CAD). In consequence, referral to neurosurgical department is delayed. Surprisingly, impairment of cardiac function and electrocardiographic (ECG) abnormalities have been demonstrated in miscellaneous neurological conditions, such as SAH [3-8] and other forms of intracerebral hemorrhage [9], acute brain injury $[10,11]$, cerebral ischemia [12], brain tumors [13], refractory status epilepticus [14], meningitis [15], intracranial hypertension and brain death [16]. First reports of the impact of central nervous system on circulation date back to the very beginning of the $19^{\text {th }}$ century, when Cushing [17] described the physiological blood pressure reaction to intracranial hypertension. More to the point, in 1990 Sato et al. [18] described an acute form of cardiomyopathy characterized by transient left ventricle (LV) apical dyskinesia ('tako-tsubo' cardiomyopathy) triggered by severe psychological distress, which provided evidence of extensive interactions between brain and heart functions, not only on structural, but also on a psychological level. Although regarded as different clinical entities, both cardiomyopathies share common pathological image of myocardial contraction-band necrosis [19], which was demonstrated in pheochromocytoma-associated cardiac dysfunction [20]. Ample scientific evidence advocates in favor of one broad spectrum of catecholamine-mediated neurovisceral damage, represented by SAH-associated cardiac dysfunction [3], tako-tsubo cardiomyopathy [21], together with neuropulmonary edema [22]. This review sought to encapsulate the up-to-date knowledge on the pathophysiology, clinical presentation and management of catecholamine-mediated neurocardiogenic injury. For this purpose, Medline and EmBase databases were searched to obtain original research articles and review papers using the following key words: neurocardiogenic injury, stress cardiomyopathy, tako-tsubo, subarachnoid hemorrhage, ECG abnormalities, catecholamine toxicity, neuropulmonary edema.

\section{Subarachnoid hemorrhage and cardiac dysfunction}

Although cardiac dysfunction was encountered in various types of cerebral pathology, it is predominantly linked to SAH. This type of cerebral incident is caused by the rupture of aneurysm at the branching of arteries located mainly on the circle of Willis. Its worldwide incidence reaches 9.1 per 100,000 person-years and is said to increase with age [23]; however, more than $50 \%$ of events occur in individuals younger than 55 years. SAH is also slightly more common in women [7]. Initially, patients exhibit a sudden acute headache, described as the most severe in a lifetime [24], along with epileptic seizures and impaired consciousness in $2 / 3$ of cases [25], which may involve both qualitative (e.g. delirium) and quantitative (e.g. coma) disturbances of cognition. Unconscious state often precludes a careful medical inquiry into the initial presentation. In the setting of emergency or cardiology department this can be the source of diagnostic errors, especially when accompanied by ECG, echocardiographic and enzymatic abnormalities typical for AMI [26].

Preliminary data on the association between ECG abnormalities and SAH were published in the middle of the $20^{\text {th }}$ century [27]. It was then suggested that those findings may actually reflect the ongoing autonomic dysfunction, secondary to cerebral insult. According to more recent studies, $69 \%$ of patients with SAH present with an abnormal admission ECG [6]. Unquestionably, a considerable portion of these findings is related to underlying $\mathrm{CAD}$ or other cardiovascular disorders, as risk factors for $\mathrm{SAH}$ and $\mathrm{CAD}$ are common (e.g. hypertension). ECG abnormalities observed in SAH are essentially related with the repolarization phase. In a cohort of 222 patients admitted to emergency department with the preliminary diagnosis of $\mathrm{SAH}$, Huang et al. [1] denoted that patients exhibited pathological Q or QS wave (8.1\%), ST-segment elevation (5\%) and depression (10.8\%), T-wave inversion (9\%), peaked upright T-wave $(2.2 \%)$ and nonspecific ST- or T-wave changes $(16.2 \%)$, as well as QT prolongation (mean QT $492 \pm 58 \mathrm{~ms}$ in non-survivors). In addition, several studies reported increased rate of prominent $U$-wave present in up to $15 \%$ of cases [1]. Worth mentioning is the lack of reciprocal lead depressions in case of observed 
ST-segment elevation. In a multivariate analysis model, it was shown that QTc and nonspecific repolarization abnormalities were independently associated with in-hospital mortality [1]. Furthermore, Coghlan et al. [28] outlined that bradycardia $(<60 \mathrm{bpm})$, relative tachycardia $(>80 \mathrm{bpm})$ and non-specific ST-T abnormalities all corresponded with increased 3-month mortality in a cohort of 588 patients undergoing aneurysm surgery. In a different study comprising 100 patients, prolonged QTc (> $460 \mathrm{~ms}$ ) was the most frequent ECG abnormality present in about $16 \%$ of patients with $\mathrm{SAH}$. Since patients with prolonged QTc were more likely to develop the elevation of cardiac troponin I (cTI), we may assume that extended QTc interval reflects neurocardiogenic injury $(\mathrm{OR}=5.5)$. However, neither QTc nor repolarization abnormalities were connected with increased mortality [29].

In this clinical setting we may also occasionally observe a specific repolarization abnormality designated as J wave [30], which was first described by Osborn in hypothermic subjects and was further reported in hypercalcemia, Brugada syndrome, vasospastic angina and, most importantly, intracranial hypertension [31]. It is defined as $1 \mathrm{~mm}$ positive notch at the end of QRS complex with duration of $\geq 10 \mathrm{~ms}$. Cellular basis for J-wave consists in potential difference between the prominent transient outward potassium current $\left(\mathrm{I}^{\mathrm{t}}\right)$ of epicardium and notably smaller $\mathrm{I}^{\mathrm{to}}$ current in the endocardial layer during phase 1 and 2 of ventricle repolarization [32]. The significance of this finding is related with frequently observed phase 2 reentrant tachyarrhythmia, sustained ventricular tachycardia and ventricular fibrillation [33] that can be induced by additional currents seen in repolarization period. The issue of arrhythmias in the acute phase of SAH requires a special consideration as it is responsible for a considerable extent of mortality. About $41 \%$ of patients without prior history of cardiovascular disorder demonstrated serious ventricular arrhythmias (Lown class 4 and 5; ventricular premature contractions with couplets or salvos, ventricular tachycardia) and 4.3\% showed life-threatening ventricular arrhythmias (torsade de pointes, ventricular flutter or fibrillation) [34]. This observation mandates constant ECG monitoring in the acute phase of SAH, especially because prompt resuscitation has a high success rate and more than $50 \%$ of survivors of in-hospital cardiac arrest manage to live independently without major neurological deficits [35].

The evidence of myocardial injury in $\mathrm{SAH}$ is frequently expressed by means of elevation of serum cardiac troponin. High prevalence of cTI elevation was documented by Tung et al. [7] who estimated that even $20 \%$ of patients suffering from SAH exhibit a cTI release of more than $1 \mu \mathrm{g} / \mathrm{L}$. In a thorough investigation by Naidech et al. [6], it was shown that increased cTI is even more frequent, since $18 \%$ of patients had an elevation of $0.5-2.0 \mu \mathrm{g} / \mathrm{L}, 14 \%-2.0-10.0 \mu \mathrm{g} / \mathrm{L}$ and $18 \%$ - more than $10.0 \mu \mathrm{g} / \mathrm{L}$. Of note, only $32 \%$ of subjects had no detectable cTI. Merely 2 out of 172 patients with elevated cTI concentration had normal clinical examination, no regional wall motion abnormalities (RWMA) and lack of ECG abnormalities, which proves the utility of cardiac troponin measurement as an indicator of neurocardiogenic injury [6]. Peak cTI predicted the occurrence of echocardiographic LV dysfunction, onset of pulmonary edema, severe hypotension requiring administration of vasopressive agents and delayed cerebral vasospasm [6]. Conversely, neurological state on admission (low neurological grade, loss of consciousness at ictus, global cerebral edema) accurately correlated with cTI release suggesting a direct link between neurological and cardiac derangement [6].

Injury of cardiomyocytes is also denoted by the surge of serum B-type natriuretic peptide (BNP) concentration, which exceeds the threshold of $100 \mathrm{ng} / \mathrm{L}$ within 3 days after ictus in about $80 \%$ of patients [36]. The release of natriuretic peptides was previously shown to correlate with LV wall stress and diastolic dysfunction encountered in chronic heart failure, but in the setting of SAH BNP did not correlate with LV filling pressure [36]. Nevertheless, prevalence of diastolic dysfunction reached $71 \%$ of all SAH subjects (impaired relaxation, pseudonormal, restrictive pattern) and was associated with older age and history of hypertension [37]. As proven by Kopelnik et al. [37], diastolic dysfunction predisposed patients to pulmonary edema, while systolic dysfunction was considerably less frequent in this clinical scenario.

Last but not least, serial echocardiographic examinations enabled to reveal a direct impairment of regional cardiac contractility in the acute phase of SAH. Consistent with the paper of Kopelnik et al. [37], $28 \%$ of patients had regional wall motion score more than 1 on at least 1 study day, suggesting RWMA [4]. In addition, $15 \%$ of patients were characterized by impaired global systolic function ( $\mathrm{LVEF}<50 \%)$. Regardless of the severity of RWMA, a partial or complete reversal within the first week of observation was seen in $66 \%$ of patients [4]. This investigation additionally provided an intrinsic evidence for the chiefly basal and 
mid-ventricular hypokinesia with a relatively low prevalence of RWMA at the apex of LV [4].

All in all, the importance of neurocardiogenic injury in SAH is related not with its frequency but with significant impact on the prognosis, as peak elevation of cTI was associated with severe disability or death at discharge (adjusted OR $=1.4$ per quintile of cTI concentration; 95\% CI 1.1-1.9, $\mathrm{p}=0.02)[6]$.

\section{Histopathology of neurocardiogenic injury in subarachnoid hemorrhage}

In this article the authors propose a novel approach to both cardiac involvement in the course of SAH and stress cardiomyopathy ("tako-tsubo'), both regarded as cardiac manifestation of catecholamine toxicity. Vast majority of data on humans endorsed sympathetic origin of the wide spectrum of neurocardiogenic injury throughout the $20^{\text {th }}$ century. Although this explanation was initially a true presumption [38], resounding evidence emerged in the form of characteristic histopathological image of catecholamine-induced lesions. In a couple of post-mortem case series $[19,39]$ it was clearly demonstrated that cerebral lesions were accompanied by a distinct type of myocardial necrosis, specifically contraction-band necrosis (coagulative myocytolysis; myofibrillar degeneration), which was first described by Selye [40]. The concurrent exposure to steroids (most importantly 2-alpha-methyl-9-alpha-fluorocortisol), certain electrolytes $\left(\mathrm{NaH}_{2} \mathrm{PO}_{4}\right)$, various hormones (vasopressin, adrenaline, insulin, L-thyroxine), and a potent stressor (restraint of an animal on a board for $15 \mathrm{~h}$ or with cold stress) created a predisposition for the development of scattered myocardial necrosis in a hyper-contracted cellular state following intracellular $\mathrm{Ca}^{++}$influx, along with irregular intracellular cross-bands, intense cytoplasmic eosinophilia and sites of petechial hemorrhage, as opposed to classic image of coagulative necrosis seen in MI. These lesions were documented to have a multifocal subendocardial predilection and were prone to early calcification [40].

These myocardial lesions were reproduced in an animal model of SAH [39, 40] and their onset was successfully prevented by pretreatment with neurogenic catecholamine inhibitors, such as reserpine (intramyocardial nerve terminals) or mecamylamine (ganglionic), which further supported the role of sympathetic nervous system. Of note, prevention of adrenomedullary secretion of epinephrine by adrenalectomy failed to exert sufficient inhibitory influence on formation of contraction-band necrosis, which may imply that direct sympathetic innervation plays a crucial role in pathogenesis of neurocardiogenic injury, as opposed to the circulating catecholamine pool.

\section{Autonomic storm}

In order to delineate the nature of neurocardiogenic injury it is indispensable to refer once again to Cushing reflex [17]. Seemingly, it plays a role in physiology maintaining adequate cerebral blood flow during rapid increase of intracranial pressure. The adaptation to intracranial hypertension is constricted to mechanical translocation and absorption of cerebrospinal fluid, which is of limited capacity to control intracranial pressure. According to Monro and Kellie [41], hypothesis deriving from incompressibility of cranium, the contents of cranium remain in the volatile balance, in which the increase in volume of one component results in a decrease of another. Provided that the volume of cerebrospinal fluid is almost constant, any lesion within cranium results in reduction of cerebral blood flow, creating a risk of cerebral ischemia. Cushing triad (hypertension, bradycardia and dyspnea/apnea) denotes a gradual and stepped autonomic response of vasomotor center to the above-mentioned situation. Initially the sympathetic center is activated, which results in the increase of norepinephrine release at nerve terminals, activating alpha- 1 adrenergic receptors, and causing rise in mean arterial pressure (MAP), which constitutes a compensation for drop in cerebral perfusion pressure. Moreover, the same sympathetic stimulation occurs in pacemaker cells and myocardium, causing a rise in heart rate and cardiac output. At this point, a parasympathetic nervous system is activated through the stimulation of carotid baroreceptors by high MAP or through direct distortion of the intracranial portion of vagal nerve. Resultant bradycardia stems from the prevalence of parasympathetic innervation of cardiac conducting system. The final consequence of brainstem compression is dysfunction of involuntary breathing, finally leading to apnea. This theoretically physiological phenomenon may occasionally become maladaptive, especially in cases of severe intracranial hypertension, leading to over-activity of sympathetic nervous system, manifested by increased norepinephrine secretion at nerve terminals in myocardium, as well as increased adrenomedullary production of epinephrine.

An additional mechanism explaining the surge in serum catecholamine level ensues in case 
of SAH or stroke. Cerebral representation of cardiac function is characterized by a hierarchy from brainstem's reticular network to cortex, where conscious awareness of heart function was proven by means of heartbeat-evoked potentials [42], predominantly in insular cortex, hypothalamus and anterior cingulate cortex [43]. Cardiac representation in insular cortex was verified by intraoperative experiments of Oppenheimer et al. [44], who demonstrated that stimulation of right insular cortex in humans triggers sympathetic response, whereas the stimulation of the left-side leads to bradycardia and depressor response. This finding was further confirmed in other studies and the structure is frequently designated as "cardunculus', in correspondence with sensory cortical motor and sensory 'homunculus' [45].

A direct insult by blood or ischemia to these cardiovascular centers may initiate a potentially deleterious response of sympathetic nervous system, which was demonstrated by Jacob et al. [46], who triggered SAH in dogs and obtained hypertension of rapid onset, extreme sinus tachycardia with coexisting arrhythmias, rise in LV pressure, and increase of coronary blood flow. Contemporary reports substantiated increased sympathetic tone and elevation of plasma catecholamine concentration in the course of SAH in humans [7, 47]. In the study by Espiner et al. [48], epinephrine and norepinephrine concentrations were increased and exhibited a gradual decrease following the incident. However, Naredi et al. [49] measured epinephrine and norepinephrine levels in non-traumatic SAH patients using a very precise isotope dilution technique and obtained disparity in terms of catecholamine concentration. Although total norepinephrine release (mostly from sympathetic nerve terminals) was 3 -fold greater in comparison to control groups (healthy individuals and patients with refractory pain), there was no statistically significant increase compared with healthy subjects in the rate of total epinephrine secretion. According to this report, elevation of norepinephrine level persisted within the first week after insult and normalized on follow-up visit after 6 months [49]. It is worth to mention that norepinephrine is efficiently removed by neuronal and extra-neuronal uptake, hence circulating norepinephrine represents merely a small portion of the total amount of this neurotransmitter. Moreover, the clinical utility of plasma catecholamine concentration is limited, since the plasma half-life of both epinephrine and norepinephrine is approximately $3 \mathrm{~min}$ [50], which implies that catecholamine concentration measured on admission to emergency department does not reflect the initial level during the onset of symptoms. In the largest, by far, study on the neurocardiogenic injury in SAH, Tung et al. [7] investigated into the possible predictors of cardiac dysfunction. It was documented that high plasma catecholamine concentration does not predict the release of cardiac troponin above the threshold of $1.0 \mu \mathrm{g} / \mathrm{L}$, which was utilized as an indicator of neurocardiogenic injury [7]. Conversely, in a multivariate logistic regression analysis, a Hunt-Hess score $>2$, female gender, larger body surface area and LV mass, lower systolic blood pressure and higher heart rate, and phenylephrine dose were independently associated with cardiac troponin elevation [7]. The relationship between Hunt-Hess score and extent of myocardial necrosis was previously demonstrated by Parekh et al. [5] and constitutes an evidence for direct link between the severity of neurological derangement and cardiac dysfunction.

\section{Catecholamine-mediated toxicity}

Cardiac dysfunction in SAH represents only one aspect of a broad spectrum of catecholamine-mediated visceral toxicity. Plasma excess of catecholamines or increased sympathetic tone are held responsible for the symptomatology of tako-tsubo cardiomyopathy, cardiac dysfunction in pheochromocytoma and a novel entity of neuropulmonary edema.

Described in 1990 by Sato [18], tako-tsubo represents a transient cardiac dysfunction concerning predominantly postmenopausal women, in which a potent stressful life event $(47 \%$ of all patients) or physical and neurological distress (in about $43 \%$ of cases) leads to retrosternal chest pain, ST-segment elevation, cardiac troponin release, markedly elevated concentration of norepinephrine [51], and transient RWMA mimicking ST-segment elevation AMI, yet resolving within days or weeks almost in all patients who survived the acute period. Coronary angiography reveals no hemodynamically significant stenosis of major coronary arteries (Table 1). Unlike SAH-associated neurocardiogenic injury, RWMA concern predominantly $L V$ apex, with a characteristic dyskinesia resembling Japanese fishing pot (tako-tsubo) on ventriculography or echocardiography. Despite distinct manifestation, stress cardiomyopathy shares the same histopathological image of contraction-band necrosis, which was proven in specimens from endomyocardial biopsy [52]. Tako-tsubo has been recently demonstrated to be a heterogeneous 
Table 1. Differentiation between neurocardiogenic injury in subarachnoid hemorrhage (SAH) and tako-tsubo cardiomyopathy.

\begin{tabular}{|c|c|c|}
\hline Clinical feature & Neurocardiogenic injury in SAH & Tako-tsubo cardiomyopathy \\
\hline Precipitating factors & $\begin{array}{l}\text { Pre-existing clinical condition (SAH, } \\
\text { intracranial hemorrhage, stroke) }\end{array}$ & $\begin{array}{l}\text { Psychological stress, less frequently } \\
\text { physical cause (e.g. SAH) }\end{array}$ \\
\hline Mechanism & $\begin{array}{l}\text { Catecholamine toxicity - } \\
\text { mainly } \uparrow \text { sympathetic tone }\end{array}$ & $\begin{array}{l}\text { Catecholamine toxicity - mainly } \uparrow \text { ad- } \\
\text { renomedullary epinephrine secretion }\end{array}$ \\
\hline Symptoms & $\begin{array}{l}\text { Neurological symptoms: acute, } \\
\text { severe headache, loss of } \\
\text { consciousness }\end{array}$ & $\begin{array}{l}\text { Angina-like retrosternal chest pain } \\
\text { Dyspnea } \\
\text { Syncope }\end{array}$ \\
\hline ECG abnormalities & $\begin{array}{l}\text { - QT prolongation } \\
\text { - T inversion } \\
\text { - ST elevation } \\
\text { - Osborne wave }\end{array}$ & $\begin{array}{l}\text { - ST-segment elevation } \\
\text { - (without reciprocal ST depression!) } \\
\text { - T wave inversion } \\
\text { - QT prolongation }\end{array}$ \\
\hline $\begin{array}{l}\text { Echocardiographic } \\
\text { findings (RWMA) }\end{array}$ & Basal, mid-ventricular RWMA & $\begin{array}{l}\text { Apical, mid-ventricular, occasionally } \\
\text { 'inverted tako-tsubo' RWMA } \\
\text { Dynamic LVOT obstruction }\end{array}$ \\
\hline Cardiac troponin level & Moderate elevation & Moderate elevation \\
\hline $\begin{array}{l}\text { Histopathological } \\
\text { findings }\end{array}$ & Contraction band necrosis & Contraction band necrosis \\
\hline Prognosis & $\begin{array}{l}\text { Dependent on the preexisting neurological } \\
\text { condition; cardiac dysfunction is worse and } \\
\text { more probable in higher Hunt-Hess grades }\end{array}$ & $\begin{array}{l}\text { Benign, transient cardiac dysfunction, } \\
\text { mortality is } 1-2 \%\end{array}$ \\
\hline
\end{tabular}

RWMA - regional wall motion abnormalities; LVOT — left ventricular outflow tract

syndrome with occasional regional wall motion abnormalities concerning mid-ventricular ('apical sparing variant') [53] and basal segments ('inverted variant') [54].

A possible clue to a different contractile pattern observed in tako-tsubo and cerebral pathology might be the pheochromocytoma-associated cardiomyopathy [20], long known to be related with excessive secretion of epinephrine and norepinephrine from the chromaffin neuroendocrine tissue of the tumor of adrenal medulla. The effect of catecholamine on myocardium results in the histological image of contraction-band necrosis and symptomatology resembles that seen in both SAH-associated cardiomyopathy and tako-tsubo, however, RWMA concern either basal [55], or apical segments of LV [56]. This variability of RWMA may be dependent on the ratio of epinephrine (prevalence of beta ${ }_{2}$ stimulation, less potent beta $_{1}$ agonist), norepinephrine (beta ${ }_{1}$ agonist) and dopamine secretion. Accordingly, high density of sympathetic innervation in the basal myocardial segments (local norepinephrine release) [57] and high concentration of norepinephrine (but not epinephrine) in SAH-associated cardiac dysfunction implies primarily sympathetic neuronal trafficking as a cause of myocardial damage in this instance [49]. Conversely, tako-tsubo with its apical dyskinesia could ensue from adrenomedullary secretion of epinephrine triggered by psychological distress. A hypothesis by Lyon et al. [58] indicated that apical catecholamine-induced myocardial stunning in tako-tsubo might, in fact, be a protective mechanism related with transient molecular switch in beta ${ }_{2}$-adrenoreceptors, from $\mathrm{G}_{\mathrm{s}}$ to $\mathrm{G}_{\mathrm{i}}$ coupling, in the event of supraphysiological plasma levels of epinephrine. This phenomenon does not occur in basal segments, since beta ${ }_{1}$-adrenoreceptor stimulation from neuronal release of norepinephrine outweighs the effects of circulating epinephrine.

From a clinical standpoint, it is vital to be aware of the possibility of concurrent lung injury in case of acute cerebrovascular incidents. Neuropulmonary edema (NPE) is manifested by rapid onset of dyspnea, tachypnea, tachycardia, basal pulmonary crackles, respiratory failure and decreased $\mathrm{PaO}_{2} / \mathrm{FiO}_{2}$ ratio in the event of cerebral incident, which represents a direct catecholamine-mediated insult to lungs [22]. Adrenergic response triggers severe pulmonary vasoconstriction, with the resultant rise in hydrostatic pressure and an increase in permeability of pulmonary capillaries [59]. Although cardiac gallop and RWMA exclude pure form of NPE, cardiac function is almost always impaired to certain extent, thus it is immensely difficult to differentiate between the NPE and pulmonary edema secondary to reduced cardiac output and increased LV end-diastolic pressure associated 
with acute heart failure. Release of cTI after SAH was verified as a predictor of acute lung injury and acute respiratory distress syndrome, regardless of neurological grade $(\log \mathrm{OR}=10.2,95 \%$ CI $3.2-31.9$, $\mathrm{p}<0.001$ ) [60]. Both disorders may then coexist, contributing to increased mortality and reflecting diffuse neurovisceral damage.

\section{Clinical implications and management}

Due to extremely low prevalence and lack of randomized trials, treatment of neurocardiogenic injury remains entirely empirical. In case of SAH-related cardiac dysfunction, it is indispensable to treat the underlying neurological condition. It is commonly believed that the "triple $\mathrm{H}$ therapy" (hypertension, hypervolemia, hemodilution) should not be withdrawn because of the threat of pulmonary edema secondary to impaired cardiac function. Neither should the aneurysmal surgery be postponed due to transient cardiac impairment. In the acute phase constant ECG monitoring is of utmost importance to early diagnose and treat ventricular arrhythmias.

However, when NPE ensues, non-invasive ventilation should be used in selected (mild cases) or patients should be intubated, sedated and appropriately ventilated with positive end-expiratory pressure $\left(<15 \mathrm{~cm} \mathrm{H}_{2} \mathrm{O}\right)$ and hemodynamically supported. Aneurysmal surgery should not be postponed for more than $12-24 \mathrm{~h}$ after the onset of symptoms. Mannitol or hypertonic saline were demonstrated, not only to normalize the elevated intracranial pressure, but also to increase the clearance of pulmonary interstitial fluid in case of NPE [61].

\section{Conclusions}

Cardiac injury, secondary to both physical and psychological insult, is mediated via common catecholamine pathway. In patients presenting with simultaneous ST-segment elevation, rise of cardiac troponin and neuropsychiatric symptoms, the decision threshold for conducting computed tomography scan before coronary angiography should be low. In case of cerebral incidents, evidence of cardiac dysfunction indicates adverse course of the underlying neurologic disorder and worsens the prognosis.

Catecholamine-induced cardio-toxicity is not only associated with structural cerebral pathology, but its involvement was also proven essential in tako-tsubo syndrome and cardiac dysfunction in pheochromocytoma. The phenomenon of NPE provides the evidence for a wide span of neurovisceral toxicity. In the light of the above-mentioned facts, the authors accentuate the need for a broad definition of neurocardiogenic injury, as the current Classification of the Cardiomyopathies by American Heart Association (2006) [62] incorporates merely tako-tsubo cardiomyopathy, defined in line with Mayo Clinic diagnostic criteria [63]. Yet, the authors conclude that this definition should be revised and extended to include all cases of neurocardiogenic injury, also these presenting without overt LV apical ballooning.

\section{Conflict of interest: none declared}

\section{References}

1. Huang CC, Huang CH, Kuo HY et al. The 12-lead electrocardiogram in patients with subarachnoid hemorrhage: Early risk prognostication. Am J Emerg Med, 2012; 30: 732-736.

2. van Gijn J, Kerr RS, Rinkel GJ. Subarachnoid haemorrhage. Lancet, 2007; 369: 306-318.

3. Davies KR, Gelb AW, Manninen PH, Boughner DR, Bisnaire D. Cardiac function in aneurysmal subarachnoid haemorrhage: A study of electrocardiographic and echocardiographic abnormalities. Br J Anaesth, 1991; 67: 58-63.

4. Banki N, Kopelnik A, Tung P et al. Prospective analysis of prevalence, distribution, and rate of recovery of left ventricular systolic dysfunction in patients with subarachnoid hemorrhage. J Neurosurg, 2006; 105: 15-20.

5. Parekh N, Venkatesh B, Cross D et al. Cardiac troponin I predicts myocardial dysfunction in aneurysmal subarachnoid hemorrhage. J Am Coll Cardiol, 2000; 36: 1328-1335.

6. Naidech AM, Kreiter KT, Janjua N et al. Cardiac troponin elevation, cardiovascular morbidity, and outcome after subarachnoid hemorrhage. Circulation, 2005; 112: 2851-2856.

7. Tung P, Kopelnik A, Banki N et al. Predictors of neurocardiogenic injury after subarachnoid hemorrhage. Stroke, 2004; 35: 548-551.

8. Kawahara E, Ikeda S, Miyahara Y, Kohno S. Role of autonomic nervous dysfunction in electrocardio-graphic abnormalities and cardiac injury in patients with acute subarachnoid hemorrhage. Circ J, 2003; 67: 753-756.

9. Qaqa AY, Suleiman A, Alsumrain M et al. Electrocardiographic abnormalities in patients presenting with intracranial parenchymal haemorrhage. Acta Cardiol, 2012; 67: 635-639.

10. Goldstein B, Toweill D, Lai S, Sonnenthal K, Kimberly B. Uncoupling of the autonomic and cardiovascular systems in acute brain injury. Am J Physiol, 1998; 275: R1287-1292.

11. Hersch C. Electrocardiographic changes in head injuries. Circulation, 1961; 23: 853-860.

12. Dimant J, Grob D. Electrocardiographic changes and myocardial damage in patients with acute cerebrovascular accidents. Stroke, 1977; 8: 448-455.

13. Koepp M, Kern A, Schmidt D. Electrocardiographic changes in patients with brain tumors. Arch Neurol, 1995; 52: 152-155.

14. Hocker S, Prasad A, Rabinstein AA. Cardiac injury in refractory status epilepticus. Epilepsia, 2013; 54: 518-522. 
15. Brander L, Weinberger D, Henzen C. Heart and brain: A case of focal myocytolysis in severe pneumococcal meningoencephalitis with review of the contemporary literature. Anaesth Intensive Care, 2003; 31: 202-207.

16. Shivalkar B, Van Loon J, Wieland W et al. Variable effects of explosive or gradual increase of intracranial pressure on myocardial structure and function. Circulation, 1993; 87: 230-239.

17. Cushing H. The blood pressure reaction of acute cerebral compression illustrated by cases of intracranial hemorrhage. Am J Med, 1903; 125: 1017-1044.

18. Sato H, Tateishi H, Uchida T et al. Tako-Tsubo-like left ventricular dysfunction due to multivessel coronary spasm. In: Kodama K, Haze K, Hori M eds. Clinical aspect of myocardial injury: From ischemia to heart failure (in Japanese). Kagakuhyoronsha Publishing Co; Tokyo 1990: 56-64.

19. Connor RC. Myocardial damage secondary to brain lesions. Am Heart J, 1969; 78: 145-148.

20. Liao WB, Liu CF, Chiang CW, Kung CT, Lee CW. Cardiovascular manifestations of pheochromocytoma. Am J Emerg Med, 2000; 18: 622-625.

21. Gianni M, Dentali F, Grandi AM et al. Apical ballooning syndrome or takotsubo cardiomyopathy: A systematic review. Eur Heart J, 2006; 27: 1523-1529.

22. Baumann A, Audibert G, McDonnell J, Mertes PM. Neurogenic pulmonary edema. Acta Anaesthesiol Scand, 2007; 51: 447-455.

23. de Rooij NK, Linn FH, van der Plas JA, Algra A, Rinkel GJ. Incidence of subarachnoid haemorrhage: A systematic review with emphasis on region, age, gender and time trends. J Neurol Neurosurg Psychiatry, 2007; 78: 1365-1372.

24. Linn FH, Wijdicks EF, van der Graaf Y et al. Prospective study of sentinel headache in aneurysmal subarachnoid haemorrhage. Lancet, 1994; 344: 590-593.

25. Brilstra EH, Rinkel GJ, Algra A, van Gijn J. Rebleeding, secondary ischemia, and timing of operation in patients with subarachnoid hemorrhage. Neurology, 2000; 55: 1656-1660.

26. Saritemur M, Akoz A, Kalkan K, Emet M. Intracranial hemorrhage with electrocardiographic abnormalities and troponin elevation. Am J Emerg Med, 2013; 31: 271 e275-277.

27. Cropp GJ, Manning GW. Electrocardiographic changes simulating myocardial ischemia and infarction associated with spontaneous intracranial hemorrhage. Circulation, 1960; 22: 25-38.

28. Coghlan LA, Hindman BJ, Bayman EO et al. Independent associations between electrocardiographic abnormalities and outcomes in patients with aneurysmal subarachnoid hemorrhage: Findings from the intraoperative hypothermia aneurysm surgery trial. Stroke, 2009; 40: 412-418.

29. Sommargren CE, Zaroff JG, Banki N, Drew BJ. Electrocardiographic repolarization abnormalities in subarachnoid hemorrhage. J Electrocardiol, 2002; 35 Suppl: 257-262.

30. Milewska A, Guzik P, Rudzka M et al. J-wave formation in patients with acute intracranial hypertension. J Electrocardiol 2009; 42: 420-423.

31. Osborn JJ. Experimental hypothermia; respiratory and blood $\mathrm{pH}$ changes in relation to cardiac function. Am J Physiol, 1953; 175: 389-398.

32. Yan GX, Antzelevitch C. Cellular basis for the electrocardiographic J wave. Circulation, 1996; 93: 372-379.

33. Shinde R, Shinde S, Makhale C et al. Occurrence of "J waves" in 12-lead ECG as a marker of acute ischemia and their cellular basis. Pacing Clin Electrophysiol, 2007; 30: 817-819.
34. Andreoli A, di Pasquale G, Pinelli G et al. Subarachnoid hemorrhage: Frequency and severity of cardiac arrhythmias. A survey of 70 cases studied in the acute phase. Stroke 1987; 18: 558-564.

35. Toussaint LG, 3rd, Friedman JA, Wijdicks EF et al. Survival of cardiac arrest after aneurysmal subarachnoid hemorrhage. Neurosurgery, 2005; 57: 25-31; discussion 25-31.

36. Meaudre E, Jego C, Kenane N et al. B-type natriuretic peptide release and left ventricular filling pressure assessed by echocardiographic study after subarachnoid hemorrhage: A prospective study in non-cardiac patients. Crit Care, 2009; 13: R76.

37. Kopelnik A, Fisher L, Miss JC et al. Prevalence and implications of diastolic dysfunction after subarachnoid hemorrhage. Neurocrit Care, 2005; 3: 132-138.

38. Cannon W. Voodoo death. Am Anthropologist 1942; 44: 169-118.

39. Greenhoot JH, Reichenbach DD. Cardiac injury and subarachnoid hemorrhage. A clinical, pathological, and physiological correlation. J Neurosurg, 1969; 30: 521-531.

40. Selye H. The chemical prevention of cardiac necrosis. Ronald Press, New York 1958.

41. Greitz D, Wirestam R, Franck A et al. Pulsatile brain movement and associated hydrodynamics studied by magnetic resonance phase imaging. The Monro-Kellie doctrine revisited. Neuroradiology, 1992; 34: 370-380.

42. Gray MA, Taggart P, Sutton PM et al. A cortical potential reflecting cardiac function. Proc Natl Acad Sci, 2007; 104: 6818-6823.

43. Critchley HD, Corfield DR, Chandler MP, Mathias CJ, Dolan RJ. Cerebral correlates of autonomic cardiovascular arousal: A functional neuroimaging investigation in humans. J Physiol, 2000; 523 (Part 1): 259-270.

44. Oppenheimer SM, Gelb A, Girvin JP, Hachinski VC. Cardiovascular effects of human insular cortex stimulation. Neurology, 1992; 42: 1727-1732.

45. Cheshire WP Jr., Saper CB. The insular cortex and cardiac response to stroke. Neurology, 2006; 66: 1296-1297.

46. Jacob WA, Van Bogaert A, De Groodt-Lasseel MH. Myocardial ultrastructure and haemodynamic reactions during experimental subarachnoid haemorrhage. J Mol Cell Cardiol, 1972; 4: 287-298.

47. Lambert G, Naredi S, Eden E, Rydenhag B, Friberg P. Sympathetic nervous activation following subarachnoid hemorrhage: Influence of intravenous clonidine. Acta Anaesthesiol Scand 2002; 46: 160-165.

48. Espiner EA, Leikis R, Ferch RD et al. The neuro-cardio-endocrine response to acute subarachnoid haemorrhage. Clin Endocrinol, 2002; 56: 629-635.

49. Naredi S, Lambert G, Eden E et al. Increased sympathetic nervous activity in patients with nontraumatic subarachnoid hemorrhage. Stroke, 2000; 31: 901-906.

50. Ferreira SH, Vane JR. Half-lives of peptides and amines in the circulation. Nature, 1967; 215: 1237-1240.

51. Kurisu S, Inoue I, Kawagoe T et al. Time course of electrocardiographic changes in patients with tako-tsubo syndrome: Comparison with acute myocardial infarction with minimal enzymatic release. Circ J, 2004; 68: 77-81.

52. Wittstein IS, Thiemann DR, Lima JA et al. Neurohumoral features of myocardial stunning due to sudden emotional stress. N Engl J Med, 2005; 352: 539-548.

53. Hurst RT, Askew JW, Reuss CS, et al. Transient midventricular ballooning syndrome: A new variant. J Am Coll Cardiol, 2006; 48: 579-583. 
54. Van de Walle SO, Gevaert SA, Gheeraert PJ, De Pauw M, Gillebert TC. Transient stress-induced cardiomyopathy with an “inverted takotsubo" contractile pattern. Mayo Clin Proc 2006; 81: 1499-1502.

55. Frustaci A, Loperfido F, Gentiloni N et al. Catecholamine-induced cardiomyopathy in multiple endocrine neoplasia. A histologic, ultrastructural, and biochemical study. Chest, 1991; 99: 382-385.

56. Subramanyam S, Kreisberg RA. Pheochromocytoma: A cause of ST-segment elevation myocardial infarction, transient left ventricular dysfunction, and takotsubo cardiomyopathy. Endocr Pract, 2012; 18: e77-e80.

57. Kawano H, Okada R, Yano K. Histological study on the distribution of autonomic nerves in the human heart. Heart Vessels, 2003; 18: 32-39.

58. Lyon AR, Rees PS, Prasad S, Poole-Wilson PA, Harding SE. Stress (Takotsubo) cardiomyopathy: A novel pathophysiological hypothesis to explain catecholamine-induced acute myocardial stunning. Nat Clin Pract Cardiovasc Med, 2008; 5: 22-29.

59. Inamasu J, Sugimoto K, Yamada $\mathrm{Y}$ et al. The role of catecholamines in the pathogenesis of neurogenic pulmonary edema associated with subarachnoid hemorrhage. Acta Neurochir, 2012; 154: 2179-2184; discussion 2184-2175.

60. Naidech AM, Bassin SL, Garg RK et al. Cardiac troponin I and acute lung injury after subarachnoid hemorrhage. Neurocrit Care, 2009; 11: 177-182.

61. Toung TJ, Chang Y, Lin J, Bhardwaj A. Increases in lung and brain water following experimental stroke: Effect of mannitol and hypertonic saline. Crit Care Med, 2005; 33: 203-208; discussion 259-260.

62. Maron BJ, Towbin JA, Thiene G et al. Contemporary definitions and classification of the cardiomyopathies: an American Heart Association Scientific Statement from the Council on Clinical Cardiology, Heart Failure and Transplantation Committee; Quality of Care and Outcomes Research and Functional Genomics and Translational Biology Interdisciplinary Working Groups; and Council on Epidemiology and Prevention. Circulation, 2006; 113 : 1807-1816.

63. Prasad A, Lerman A, Rihal CS. Apical ballooning syndrome (Tako-Tsubo or stress cardiomyopathy): A mimic of acute myocardial infarction. Am Heart J, 2008; 155: 408-417. 